\title{
SEXUAL BEHAVIOR OF WOMEN WITH BREAST CANCER AND IMPACT ON THE COUPLE'S LIFE
}

\author{
Мауага M. P. Carvalho1, Ruffo de Freitas Júnior ${ }^{1,2}$ \\ ${ }^{1}$ Universidade Federal de Goiás (UFG) - Goiânia (GO), Brazil. \\ ${ }^{2}$ Centro Avançado de Diagnóstico da Mama, UFG - Goiânia (GO), Brazil.
}

Sexuality is experienced by individuals from their birth until old age, and is related to their history and culture. Sex, a constituent factor of human physical and emotional health, derives from an intrinsic characteristic of humans regardless of their social status, and changes according to the cultural and historical context. Sexual activity is one of the main pillars of individuals' sexuality and well-being, and not only associated with reproduction. The emotional aspects related to sexual dysfunction in people with cancer are common and frequent, and may occur since the diagnosis. Breast cancer is the second most common type of cancer worldwide, the most frequent in women, and the leading cause of death in patients with malignant neoplasms. In the state of Goiás (Brazil), the estimated number of new breast cancer cases in 2017-2018 is 49.51 per 100,000 women. In general, breast cancer tumors present morphological, clinical, and biological heterogeneity, due to different histological grades as well as molecular differences. Breast cancer may have multifactorial impact on the quality of life, sexual relationships, and, consequently, marital relationships of patients. Furthermore, the disease can interfere with patients adherence to endocrine therapy. Breast cancer staging may determine the level of sexual dysfunction in women. At early stages, the most reported symptoms are loss of libido and lack of sexual desire, and at later stages, loss of interest in sex and pain during sexual intercourse. Partners' support and understanding are fundamental for women's psychological acceptance of the disease. However, the partners of women with breast cancer also have psychological impacts, which can lead to premature ejaculation, erectile dysfunction, and depression and anxiety disorders. Objective: Verify whether the sexuality of both women diagnosed with breast cancer and their partners is affected and the possible causes. Methodology: This is a narrative review. The literature review was based on the strategic planning for the search that included the following steps: 1. Constructing a guiding question that explains the interest of the research; 2. Identifying the elements of the research (variables, studied populations, comparisons); 3. Constructing search strategies using keywords (descriptors); 4. Applying the search strategies developed for this study to the articles found in the PubMed Central (PMC) at the U.S. National Institutes of Health's National Library of Medicine (NIH/NLM); 5. Defining search filters such as articles published in the last 5 years, research carried out with humans, with adults; 6 . Exclusion criteria: homosexual couples, quality of life, breast cancer in men, and studies that assessed sexuality in single women. The search strategy applied was: cancer treatment OR female breast cancer AND sexuality OR sexual health AND partner OR couple. The filters were: last 5 years and studies with humans. After applying the search strategy and identifying the studies of interest, the first selection of the material was performed analyzing the titles, followed by the selection of the abstracts, and only at the last level of selection the complete articles were identified and read to compose this narrative review. Results: In this narrative review, 150 articles were initially collected and examined. After applying the exclusion criteria, 54 were selected. The authors of the articles included in this review unanimously reported that women diagnosed with breast cancer had impairments regarding their sexual performance, due to physiological, therapeutical, and/or emotional changes associated with the neoplasia since the diagnosis. Breast cancer treatment may cause a decline in couples' quality of sexual life. Depression, anxiety, conflicts in the relationship, and low self-esteem can intensify sexual dysfunction in women treated for breast cancer, and this impairment may last many years after the treatment. Mastectomy may substantially aggravate sexual intercourse during and after treatment due to sequels, which may affect couples' relationships. Conclusion: Current therapeutic approaches for breast cancer can considerably affect women's sexual performance due to their adverse effects. Therefore, the negative aspects of dealing with psychological and emotional suffering can be potentially aggravated in women diagnosed with breast cancer. Aiming to minimize this suffering, we propose that psychological and medical interventions be offered to women with breast cancer and their partners, both individually or to the couple, during and after the treatment. However, it is of paramount importance that healthcare professionals be attentive to the slightest signs of sexual dysfunction and recommend psychological follow-up. 